\section{AUTOPOIESIS, BUCLES, EMERGENCIA, VARIEDADES TOPOLÓGICAS Y UNA CONJETURA SOBRE LA CONSCIENCIA HUMANA}

\author{
José A. Sarmiento Campos \\ Universidad de Vigo \\ Rúa San Rosendo n. ${ }^{\circ} 11,3 .^{\circ} \mathrm{A}$. \\ 32001 Ourense \\ sarmiento@uvigo.es
}

\section{AUTOPOIESIS, FEEDBACK LOOPS, EMERGENCY, TOPOLOGICAL MANIFOLDS AND A HUMAN CONSCIOUSNESS CONJECTURE}

\begin{abstract}
This reflection aims to be a plausible approach to the concept of consciousness and it proposes a theory to explain its emergency. This theoretical approach integrates some of the contributions that different authors have made to science. Some of these contributions are examples of complexity in themselves (such as the concept of system, autopoiesis, emergency, cybernetic); some others are in the scope of mathematics (the concept of $n$-dimensional topological manifold) and even some of them are related to biology (neural networks, cortical layers or columnar organization of the neocortex). All of them can be integrated in a theory, which we consider epistemologically solid and could provide an answer to questions such as the emergency of human and social consciousness.
\end{abstract}

KEY WORDS: Complexity, consciousness, emergence, dimension, neocortex.

UNA INTRODUCCIÓN: DEL SISTEMA A LA EMERGENCIA DE LA AUTOPOIESIS

La teoria de sistemas que desarrolla Von Bertalanffy en el año 1940 como una reflexión sobre la biología se expande en las más variadas direcciones y va a poner en una situación crítica los principios del conexionismo reduccionista. Este concepto, que ha dado lugar a numerosas definiciones, como las recogidas por Nemiche (2004: 10), incluye tanto a los sistemas abiertos como cerrados, todos los elementos constituyentes del mundo forman un conjunto interrelacionado de partes que integran un todo organizado.

Los organismos vivos, las galaxias, los átomos, los ecosistemas... se pueden entender como un conjunto de elemen-
RESUMEN: La presente reflexión pretende ser un acercamiento plausible al concepto de consciencia y propone una explicación a su surgimiento. Varias de las aportaciones que diversos autores han hecho a la ciencia y que conforman el paradigma de la complejidad, como son los conceptos de sistema, autopoiesis, emergencia, cibernética de primer y segundo orden o estado crítico; junto con otros de naturaleza matemática como las variedades topológicas n-dimensionales y aun otros procedentes de la biología como redes neuronales, capas corticales o distribución columnar de la neocorteza, se pueden integrar en un constructo, que consideramos epistemológicamente sólido, que podría dar una respuesta a cuestiones como la emergencia de la consciencia humana y también, probablemente, social.

PALABRAS CLAVE: Complejidad, consciencia, emergencia, dimensión, neocórtex.

tos constituidos en una autoorganización que posibilita la existencia del sistema (Bertalanffy, 1976).

Se podria hablar de tres características estructurales básicas de todo sistema: uno, los elementos que lo componen, dos, las relaciones entre esos elementos, y tres, los límites del propio sistema que determinan que elementos pertenecen al mismo y cuáles no (Martínez y Requena, 1986: 58).

Mientras que los sistemas cerrados presentan unos límites muy claros que actúan como fronteras impidiendo la interacción con cualquier elemento ajeno a sí mismo, la naturaleza de los sistemas considerados abiertos posibilita la interacción y relación con elementos ajenos. Los referidos sistemas abiertos provienen originariamente 
del campo de la Termodinámica, como necesidad teórica para permitir circunscribir mediante realimentación negativa el campo de aplicación del segundo principio, que requiere la noción de sistema cerrado. A partir de la definición anterior fue posible considerar cierto número de sistemas, sobre todo los vivos, como sistemas cuya estructura y existencia dependen de un aporte exterior y no sólo material-energético, sino también organizativo y de información. Esto estableció un puente entre la termodinámica y la ciencia de lo vivo que permitió a Maturana y Varela (1996: 40), en relación con los sistemas vivos, definir conceptos como organización, patrón y autopoiesis, conceptos que han significado un importante salto cualitativo en la forma de entender los fenómenos como sistemas complejos.

De la teoría sistémica se derivan los conceptos de emergencia y de límite o frontera. Todo sistema a partir de su propia estructura organizativa, desarrolla un conjunto de propiedades que no es posible explicar a partir del análisis aislado de las partes que lo forman y que se denominan emergencias. Esa misma estructura organizativa posibilita el desarrollo de determinadas propiedades que constituyen los límites o fronteras del sistema.

La configuración de las relaciones entre los componentes del sistema determina las propias caracteristicas esenciales, lo que Maturana y Varela (1996: 42) denominan patrón de organización. En el caso de los sistemas vivos este patrón comporta la existencia de la autopoiesis como una emergencia. Autopoiesis como capacidad del sistema para mantenerse o producirse a sí mismo a partir de una relación dialógica con el medio.

\section{Cibernética y holística: PROCESOS de ReAlimentación}

La palabra Cibernética, según el Diccionario de la Real Academia (2001: 370), deriva del griego "Kybernetes", aparece por primera vez con Platón, y fue usada en el siglo XIX por André Marie Ampere para referirse a los modos de gobierno, pero es Wiener (1948) quien acuña el término "cibernética" para dar nacimiento a la ciencia del control y la comunicación en el animal y la máquina. Los trabajos en este campo se remontan a los años cuarenta del siglo XX, a los estudios sobre retroalimentación y causalidad circular desarrollados por Von Neuman y Wiener (Heims, 1987). Preocupados por la cantidad de información codificada como una señal que podía pasar por un canal, Wiener y Shanon comenzarán a trabajar en el campo de la teoria de la información. La profundización en estos estudios por parte de McCulloch y Ashby (Blanco, 2005) cristalizará en una propuesta integradora, la cibernética del cerebro, en la que se aplicarán los modelos de la teoría de la información a la cognición humana. En este grupo se encontraban científicos de diferentes campos (matemáticos, ingenieros, neurocientíficos, científicos sociales) y además de los cinco apuntados anteriormente también formaban parte de dicho grupo Gregory Bateson y Heinz Von Foerster (Bonil, 2003: 92-95). Wiener y Bateson se dedicaron a buscar siempre el patrón común que está detrás de los fenómenos vivos, describiéndolos holísticamente. La consideración de la red como patrón organizativo de los sistemas es el punto de partida de las aportaciones de la cibernética y la teoría de la información a los estudios de los sistemas vivos. A lo largo de los años sesenta, Bateson desarrolló un concepto de mente como fenómeno sistémico, mientras que Neumann creó el primer modelo de ordenador digital.

A partir las diversas teorías desarrolladas por los autores citados en los párrafos anteriores se puede entender cualquier sistema como una red no lineal donde la información circula a través de sus mallas, pudiendo formar ciclos. Estos bucles tienen efectos de autorregulación del propio sistema. Los bucles de realimentación se pueden entender como disposiciones circulares de elementos que están conectados causalmente, de manera que una fluctuación inicial circula por todas las mallas en una cadena continua de multicausas y multiefectos que a la vez son causas de efectos posteriores, llegando a realimentar al nódulo inicial, haciéndolo causa de sí mismo, como se puede ver en la figura 1.

Matemáticamente un bucle de realimentación corresponde a un proceso iterativo, que consiste en una función que opera reiteradamente sobre sí misma. Los estudios de funciones iterativas permiten profundizar en la comprensión de los bucles retroactivos o recursivos. Los fenómenos de recursividad pueden tener una perspectiva negativa o positiva. Un bucle recursivo positivo tiende a aumentar el efecto de la fluctuación inicial, mientras que un bucle recursivo negativo tiende a minimizarla. Wiener 


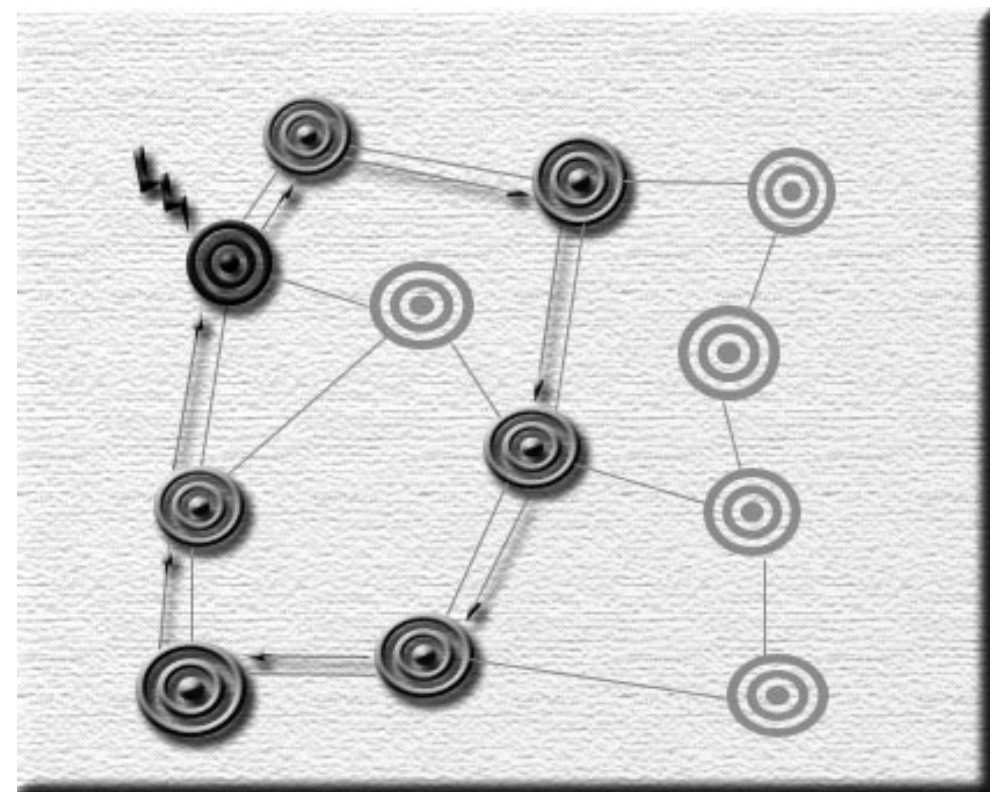

Elaboración propia.

(1956) enfatizó la importancia de la retroalimentación como modelo no sólo de los organismos vivos, sino también para explicar el comportamiento de los sistemas sociales.

En un sistema vivo la autoorganización es un elemento fundamental, ya que la evolución del propio sistema parte de procesos autoorganizativos que son generados a partir de retroacciones entre los elementos que forman el sistema y entre el propio sistema y el medio (Johnson, 2003: 67). En la actualidad se considera que detrás de los procesos de autoorganización hay un gran potencial creativo, puesto que, como se ha visto, un bucle recursivo puede llevar a la aparición espontánea de nuevas estructuras y de nuevos comportamientos. Concluyendo con las palabras de Bonil puede decirse que:

"nos encontramos delante de unos modelos explicativos de procesos donde confluyen multitud de causas y efectos en relaciones retroactivas que generan procesos autoorganizadores que permiten explicar la evolución de los sistemas vivos en términos de diálogo entre linealidad y no linealidad a partir de la circulación de la información". Bonil (2003: 94).

\section{EL AZAR O LA IMPOSICIÓN SISTÉMICA}

Entre las diferentes disciplinas que se han ocupado del estudio del azar y la indeterminación destaca el impacto que ha tenido la teoría cuántica en el campo de la física y el estudio de los sistemas dinámicos en el campo de las Matemáticas.

La Interpretación de Copenhague u ortodoxa de la teoría cuántica, expuesta por Bohr en la capital danesa en el transcurso de una serie de conferencias durante la década de los 20 del pasado siglo, consiste en entender que las entidades subatómicas, como por ejemplo los electrones, no poseen existencia real; existen en un espacio probabilistico de muchos estados superpuestos posibles que finalmente son forzados a un estado único por el acto de la observación. Los electrones y los fotones se pueden encontrar como ondas o partículas, según la forma en que se observen experimentalmente (Horgan, 1998). Esto integra al azar en el campo de la física. En este sentido Prigogine (1997) destaca el papel esencial de la probabilidad y el nuevo significado del azar que invalida en una amplia gama de fenómenos las predicciones de los esquemas deterministas.

ARBOR CLXXXV 738 julio-agosto [2009] 871-878 ISSN: 0210-1963 
Al mismo tiempo que la Física se abría a lo complejo a través de la teoría cuántica, en el campo de las matemáticas se estaba desarrollando la teoría de los sistemas dinámicos como un conjunto de conocimientos generados a partir de las investigaciones en los campos de las iteraciones, la geometría fractal, la topología y la teoría del caos.

Finalmente, no se puede hablar de azar, al menos en los sistemas biológicos, sin hacer referencia al pensamiento del biólogo Monod (1989). En su obra los términos azar y necesidad aparecen como emergencia, ya tratada anteriormente, y teleonomía, respectivamente. Contempla la emergencia como la propiedad de reproducir y multiplicar estructuras ordenadas muy complejas, y de permitir la creación evolutiva de estructuras de complejidad creciente. Por su parte, la teleonomía da respuesta a la finalidad de la estructuración, organización y condiciones del ser vivo, a la supervivencia del individuo y de la especie.

\section{EL CONCEPTO DE IRREVERSIBILIDAD}

Autores como Gell-Mann (1995), Lewin (1995) o Prigogine (1997) han incorporado los estudios sobre irreversibiliad a sus propios modelos explicativos de los seres vivos desde una perspectiva de la complejidad. Desde el campo de la Termodinámica surge la entropía como un parámetro fundamental en el estudio de los procesos de regulación de los sistemas vivos. Este término hace referencia a una función de estado "S" que caracteriza el estado de "desorden" de un sistema. Este concepto amplió su influencia más allá de la Física y así en la Teoría de la comunicación mide la incertidumbre de la naturaleza de un mensaje dado a partir de la que le antecede. Entropía no es un concepto absoluto ya que mide variación entre dos estados diferentes de un sistema. Durante la evolución de un sistema aislado, su entropía aumenta excepto en sistemas autoorganizados o autopoiéticos, con propiedades autoconstructivas como en los seres vivos (Maturana y Varela, 1980: 24).

Para realizar los procesos reguladores, los sistemas vivos aparecen como espacios de diálogo constante entre estructura y componentes, flujo constante de materia y energía y funciones especificas. La intensidad de las fluctuaciones puede producir aumentos de entropía que al llevar al sistema a un alto grado de desorden pueden hacerlo derivar en una estructura disipativa (Prigogine, 1997), una situación límite (Gell-Mann, 1995) o acercarlo al límite del caos (Lewin, 1995), tres conceptos que tienen un significado equivalente y sirven para expresar el momento en el que el sistema llega a un estado crítico.

El aumento de entropía hace que el proceso tenga carácter de irreversible. Este aspecto hace imposible que el sistema retorne a un estado anterior y orienta su evolución cara al futuro, tomando fuerza la idea de indeterminación.

Las perspectivas anteriores logran traspasar sus dominios disciplinares y desarrollan un entramado teórico altamente plástico que permite transversalizar las ciencias, ir y venir desde las ciencias físicas hasta las ciencias humanas o sociales. El paradigma de la complejidad estudia cómo desde la materia inerte hasta la materia viva y hasta lo social, se efectúa una transferencia que pretende alcanzar cierto orden aumentando su complejidad. Lo vivo y lo social tienen en común que son sistemas abiertos y complejos.

En este punto y tratando la transversalización de la ciencia y entre sistemas es necesario hacer referencia a la teoria que subyace en toda la obra de Luhmann y al tratamiento que hace de la complejidad. Inicialmente la complejidad aparecía como el conjunto de posibilidades de encontrar un patrón 0 un sentido en un conjunto de vivencias o acciones. Posteriormente el referente pasó de ser el individuo a ser el sistema y las posibles selecciones que puede efectuar ante un conjunto de posibilidades. La complejidad obliga a una toma de decisiones, no sobre todas las posibilidades existentes (inasibles a un observador) sino a las que puede hacer frente el foco del observador. Para Luhmann (1998) la complejidad es una cualidad que se da en la relación observador-observado, además existe circularidad entre la forma en que se define complejidad y la actividad autopoiética del sistema desde el que se hace la selección y consecuentemente desde donde se definen los límites de los complejo que queda "al otro lado". La complejidad se construye circularmente, autorreferencialmente, pues es este proceso autorreferencial el que permite establecer el gradiente de complejidad.

Conforme al pensamiento de Morin (1996) los sistemas físicos, vivientes y sociales, son una unidad de unidades o una unidad múltiple, constituyéndose en paradoja. Desde el punto de vista del todo, cada sistema es uno y homogé- 
neo; desde la perspectiva de sus componentes, los sistemas son múltiples, diversos y poco homogéneos. No debemos caer en el reduccionismo del todo a las partes, ni de las partes al todo, ni de lo uno a lo múltiple, ni de lo múltiple a lo uno, sino que es preciso contemplar todas estas posibilidades en relación complementaria y antagonista.

Esta visión ecosistémica de interrelación e interdependencia mutua sitúa también al sujeto dentro de esta realidad de imbricaciones como sistema que percibe a otros sistemas, pero que a la vez es percibido y concebido por éstos. Von Foerster (1991) al referirse a la cibernética de segundo orden pretendía superar visiones reduccionistas y simplificadoras que aislaban al sujeto de su objeto de conocimiento, creyendo que de esta forma se garantizaba la objetividad de la ciencia. Von Foerster entendía que no existen ecosistemas sin sujetos, el sujeto que investiga forma parte de su propia investigación desde un proceso cibernético de segundo orden.

\section{UNA BREVE PAUSA: ACERCAMIENTO TANGENCIAL A LA GEOMETRÍA Y LA TOPOLOGÍA}

Echemos mano de la abstracción matemática imaginando un mundo plano. Entenderemos como tal un espacio bidimensional formado por infinidad de puntos definidos por duplas de valores, uno para cada dimensión de las que componen ese mundo. Los objetos que ocupan ese espacio estarán sujetos a la naturaleza impuesta por las dos dimensiones y cualquier observador perteneciente a ese mundo estaría también constreñido por el propio contexto del que forma parte, estaría sometido a la circularidad de los bucles relacionales que se pudieran establecer entre los elementos que conformaran ese mundo plano. Estaría atrapado dentro de un proceso cibernético de segundo orden.

¿Cómo podría un sujeto bidimensional ser observador de su mundo? Saliendo del mismo gracias a la emergencia de una nueva dimensión, es decir convirtiéndose en un observador de tres dimensiones.

Pero podría darse el caso de que exista un mundo de tres dimensiones espaciales, como el nuestro, en el que ciertas estructuras se comporten como mundos planos, estructuras funcionalmente bidimensionales en un mundo tridimensional. A modo de ejemplo podríamos citar la superficie de nuestro propio planeta proyectada en un mapamundi, en un plano. Lo que se pueda representar en una superficie plana será de naturaleza bidimensional y si sus elementos constitutivos son de naturaleza autopoiética estarán siempre dentro de un proceso cibernético de segundo orden. Dejémoslo por ahora ahí.

\section{LA CONSCIENCIA}

Siguiendo la argumentación que hace Koch (2005: 38-39), podríamos establecer la consciencia como el nexo de unión entre mente y cuerpo que se materializa en la experiencia subjetiva y que no es exclusiva de los seres humanos. Una definición un tanto difusa pero que nos sirve como punto de partida.

De acuerdo con el citado autor entendemos que para que las criaturas biológicas, autoorganizativas, experimenten algo, es necesaria y suficiente la actividad cerebral, sin otro aditamento.

Centrémonos ahora en la actividad cerebral del ser humano, o más específicamente en la organización o estructura que propicia dicha actividad.

En los manuales de Neurociencia a menudo se habla de circuitos nerviosos o neuronales como conjunción de agrupaciones neuronales, con características funcionales diferentes, que realizan funciones mucho más complejas que las que podrían realizar un tipo único de neurona (Delgado, Ferrús, Mora y Rubia. 1998). El cerebro se nos presenta como una estructura reticular o entramado de células cuya misión es la transmisión de estímulos mediante la comunicación entre ellas (Portellano, 2005). No obstante, tanto circuitos como redes pueden ser representados en planos, son variedades bidimensionales.

La consciencia humana tiene como soporte las redes neuronales anteriormente aludidas, pero para emerger tiene que trascender esa bidimensionalidad de la red y adquirir otra nueva dimensión, ha de modificar la estructura reticular para alcanzar esa tercera dimensión que permita reconocerse como individuo. 


\section{LA ESTRUCTURA COLUMNAR DEL NEOCÓRTEX}

Las tinciones argénticas muestran que el neocórtex 0 isocórtex, la estructura filogenéticamente más reciente del cerebro humano, está constituido por seis capas celulares, criterio citoarquitectónico que ha servido, entre otros, para establecer tres clases: córtex agranular, córtex hipergranular (áreas sensitivas y sensoriales) y córtex laminar (áreas asociativas).

Arteaga y Pimienta (2004) hablan de organización horizontal y vertical de la corteza cerebral. Mientras que el patrón de organización horizontal se refiere a la disposición en capas paralelas a la superficie cortical, el patrón vertical describe agrupamientos celulares, ortogonales a la superficie, que han recibido el nombre de columnas.

Mountcastle (2003) estableció la unidad básica de la operación cortical en la minicolumna, una estructura vertical de entre ochenta y cien neuronas distribuidas a lo largo de todas las capas corticales a excepción de la primera y conteniendo todos los tipos de células de la corteza, constituyendo unidades de procesamiento complejo.

\section{UNA CONJETURA UNIFICANDO LO ANTERIOR}

El elemento humano participa en tres sistemas diferentes y autoorganizados como son el sistema biológico, el sistema de conocimiento o cognitivo y el sistema social. Esta triple pertenencia provoca una extremada complejidad y se concreta como representación de la máquina no trivial por excelencia (Von Foerster, 1991).

La idea anterior aparece en la obra de Luhmann $(1991,1996$, 1998) como un concepto básico. Según este autor existen cuatro grandes tipos de sistemas: las máquinas, los organismos, los sistemas psíquicos y los sistemas sociales. Sólo tres de ellos son autopoiéticos: organismos vivos, sistemas psíquicos y sistemas sociales (véase figura 2) y sólo los dos últimos pueden ser comprendidos y a su vez comprender (Luhmann, 1991).

Complejidad, sistema, autopoiesis, emergencia, cibernética de primer y segundo orden, azar, estados críticos, variedades topológicas y organización neuronal son conceptos que
Figura 2. Tipos de sistemas según Luhmann.

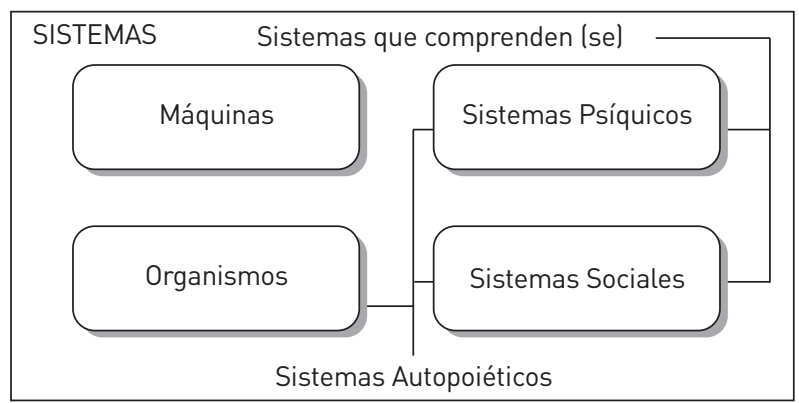

Elaboración propia.

interpretamos como indicios y que nos animan a formular en cinco pasos una, en primera instancia, conjetura sobre la emergencia de la consciencia que habrá de someterse a profundas investigaciones y concienzudos contrastes.

1. ${ }^{\circ}$ El ser humano participa de tres sistemas autopoiéticos, dos intrínsecos y uno extrínseco.

2. ${ }^{\circ} \quad$ El primer sistema es de naturaleza biológica y su emergencia es la vida. Sus elementos constitutivos (células) establecen relaciones entre ellos, cada vez más complejas. El mayor grado de complejidad aparece en las células que componen el cerebro, pero la estructura que conforman, excepto en la neocorteza, es una variedad bidimensional. Puede ser representada en un plano.

3. Cuando la complejidad de un sistema autopoiético llega a un estado crítico emerge una nueva dimensión. La red neuronal humana como variedad bidimensional llega a su estado crítico en la neocorteza, emergiendo, gracias a su disposición columnar, una nueva dimensión (variedad tridimensional) y con ella la consciencia, que todavía forma parte del primer sistema autopoiético humano.

4. El segundo sistema es de naturaleza simbólica y su emergencia es el pensamiento. La variedad tridimensional del cerebro humano alcanza su estado crítico y emerge una nueva variedad con una cuarta dimensión: el lenguaje.

5. El tercer sistema autopoiético, extrínseco, es de naturaleza comunicativa y su emergencia es la sociedad. El ser humano como elemento-sustrato de este tercer sistema está sujeto a participar siempre en procesos 
cibernéticos de segundo orden y en su naturaleza no figura la posibilidad de acceder a una nueva variedad pentadimensional. Dicha posibilidad sólo la alcanzará la sociedad cuando llegue a su estado crítico.

\section{Algunas CONSIDERACIONES finales}

Se asume la relación como una situación que se da entre elementos físicos, cognitivos o sociales cuando por alguna circunstancia están unidos de manera real o imaginaria, es decir, cuando el sujeto es capaz de identificar límites comunes entre dichos elementos.

El sujeto humano puede percibir otro elemento cuando identifica sus límites. Puede percibir varios elementos cuando discrimina unos de otros. La facultad de identificar relaciones entre elementos le permite distinguir elementos de elementos, es decir, conjuntos, es decir, estructuras. Recursividad, metaestructuras, complejidad. En el caso de no existir una unidad de propósito en la estructura, ésta sólo se limitaría al hecho de ser. La estructura es, no evoluciona, es forma en el espacio. Es intemporal. La estructura temporal, que evoluciona y de esta manera crea el tiempo, deviene en sistema. Sistema compuesto de elementos y relaciones entre elementos, relaciones posibles y probables que hacen que la entropia aumente, que el desorden aumente a lo largo de la evolución del sistema y lleguen a estados críticos, excepto en los sistemas que se autocontruyen, autorreparan y convierten ese punto crítco en una nueva dimensión, como los sistemas vivos.

Al detenerse en los sistemas vivos, se constata que existe, al menos, un metasistema: el ser humano. El sujeto humano identifica sus límites y toma autoconciencia de sí, y al mismo tiempo de los otros que son como él. Por una parte se siente sistema individual y por otra se sabe parte de otro un nivel más allá, formado por elementos de su misma naturaleza: el sistema social.

Conciencia de sí, es decir realimentación o autorreferencia, concepto clave y pilar de la cibernética, pirueta de la naturaleza que propicia la existencia de la autopoiesis, que es decir tanto como la posibilidad de generar sistemas y regenerar el propio sistema, de modificar en cierta medida su propio proceso entrópico, de propiciar una vida que mantiene la entropía en un tiempo lento.

Elemento humano, sistema humano, metasistema humano. Tres sistemas diferenciados que interactúan y emergen en un elemento-sistema consciente de su existencia y de la existencia de elementos-sistemas de su misma naturaleza. Un sistema biológico que se desarrolla según su constitución física y los procesos inherentes al propio sistema.Un sistema cognitivo que se desarrolla sobre estructuras simbólicas formales o pseudoformales y que necesita del soporte sistémico de nivel inferior, de sus dimensiones. Un sistema social del que forma parte como elemento.

Del proceso de desarrollo de cada uno de los sistemas "humanos" retorna, resulta o emerge una característica propia (unidad de propósito) e inherente a cada uno de ellos y que se concreta en la vida para el sistema biológico y el pensamiento en el sistema cognitivo. El individuo humano como elemento constitutivo del sistema social no tiene capacidad para aprehender la emergencia de dicho sistema, ya que ésta se sitúa un nivel por encima del cognitivo del individuo humano. Desde la naturaleza cognitiva sólo podemos conocer los elementos y las relaciones que constituyen el sistema social y que se concretan en individuos humanos y relaciones de comunicación, podemos conocer el nombre: cultura, pero no su esencia.

\section{BIBLIOGRAFÍA}

Recibido: 14 de enero de 2009

Aceptado: 27 de febrero de 2009
Arteaga, G. y Pimienta, H. (2004): "Sobre la organización columnar de la corteza cerebral", Revista colombiana de psiquiatría, XXXIII(5), 76-101.
Bertalanffy, L. (1976): Teoría general de los sistemas: fundamentos, desarrollo, aplicaciones, Madrid, Fondo de Cultura Económico de España, S.L.

Blanco, C. J. (2005): "El constructivismo biológico ¿Una alternativa al realis- 
mo?", Cinta de Moebio, n. 22, marzo, Facultad de Ciencias Sociales, Universidad de Chile. Consultado el 20 de abril en: http://www.moebio.uchile. $\mathrm{cl} / 22 /$ blanco.htm.

Bonil, J. (2003): La recerca avaluativa d'un programa de l'assignatura de didàctica de de les ciències experimentals dissenyat prenent com a marc teòric el paradigma de la complexitat: orientacions per al canvi (tesis doctoral), Facultat d'Educació. Universidad Autónoma de Barcelona.

Delgado, J.; Ferrús, A.; Mora, F. y Rubia, F. (1998): Manual de neurociencia, Madrid, Sintesis.

Gell-Mann, M. (1995): El quark y el jaguar, aventuras en lo simple y lo complejo, Barcelona, Tusquets.

Heims, S. J. (1987): Jon Von Newmann and Norbert Wiener: From mathematics to the Technologies of life and death, Cambridge, MIT Press.

Horgan, J. (1998): El fin de la ciencia, los límites del conocimiento en el declive de la era científica, Barcelona, Paidós.
Jonson, S. (2003): Sistemas emergentes, o qué tienen en común hormigas, ciudades y software, México DF, Turner, Fondo de Cultura Económica.

Koch, C. (2005): La consciencia, una aproximación neurobiológica, BarceIona, Ariel.

Lewin, R. (1995): Complejidad, el caos como generador del orden, Barcelona, Tusquets.

Luhmann, N. (1998): Complejidad y modernidad, Madrid, Editorial Trotta, S.A.

Luhmann, N. (1991): Sistemas sociales. Lineamientos para una teoría general, México, Alianza editorial/Universidad Iberoamericana.

Luhmann, N. (1996): Teoría de la sociedad y pedagogía, Barcelona, Paidós.

Martínez, S. y Requena, A. (1986): Dinámica de sistemas. 1. Simulación porordenador. 2. Modelos, Madrid, Alianza (2 vols.).

Maturana, H. y Varela, F. (1996): El árbol del conocimiento, Barcelona, Debate.

Maturana, H. L. y Varela, F. G. (1980): Autopoiesis and cognition: The realization of living, Dordrecht, D. Reídle.
Monod, J. (1989): El azar y la necesidad, Barcelona, Tusquets.

Moreau, J. (1971): Spinoza et le spinozisme, Paris, PUF.

Mountcastle, V. (2003): "Introducción", Cerebral Cortex, Jan, 13, 1047-3211.

Morin, E. (1996): Introducción al pensamiento complejo, Barcelona, Gedisa.

Nemiche, M. (2004): Un modelo sistémico de evolución social dual, Valencia, Servicio de publicaciones Universidad de Valencia.

Portellano, J. A. (2005): Introducción a la neuropsicología, Madrid, McGrawHill.

Prigogine, I. (1997): ¿Tan solo una ilusión? Una exploración del caos al orden, Barcelona, Tusquets.

Von Foerster, H. (1991): Las semillas de la cibernética, Barcelona, Gedisa.

Wiener, N. (1948): Cybernetics, or Control and Communication in the Animal and the Machine, Cambridge, MIT Press.

Wiener, N. (1956): The Human Use of Human Beings - Cybernetics and Society, Garden City, NY, Doubleday. 